\title{
Les Patriotes et le gouvernement responsable dans les années
}

\section{0}

\section{F. Murray Greenwood}

Volume 33, numéro 1, juin 1979

URI : https://id.erudit.org/iderudit/303749ar

DOI : https://doi.org/10.7202/303749ar

Aller au sommaire du numéro

Éditeur(s)

Institut d'histoire de l'Amérique française

ISSN

0035-2357 (imprimé)

1492-1383 (numérique)

Découvrir la revue

Citer cet article

Greenwood, F. M. (1979). Les Patriotes et le gouvernement responsable dans les années 1830. Revue d'histoire de l'Amérique française, 33(1), 25-37.

https://doi.org/10.7202/303749ar d'utilisation que vous pouvez consulter en ligne.

https://apropos.erudit.org/fr/usagers/politique-dutilisation/ 


\title{
LES PATRIOTES \\ ET LE GOUVERNEMENT RESPONSABLE DANS LES ANNÉES 1830
}

\author{
F. Murray GreenwOOD \\ Département d'bistoire \\ University of British Columbia
}

On a parfois laissé entendre que les Patriotes ne comprenaient guère le système ministériel du gouvernement responsable, qui était en pleine évolution en Grande-Bretagne pendant les années $1830^{1}$. Et l'on tire habituellement, de cette constatation, la conséquence qu'une telle ignorance écartait toute possibilité d'une issue pacifique à l'impasse constitutionnelle dans laquelle on se débattait à l'époque ${ }^{2}$. Certains, par ailleurs, ont mis en lumière l'une ou l'autre déclaration individuelle de patriotes pour démontrer que le concept n'en était pas inconnu. Ces auteurs n'ont fait voir clairement, toutefois, ni le degré de compréhension qu'avaient les Patriotes de la responsabilité ministérielle, ni leur appréciation des avantages et des faiblesses du système ${ }^{3}$.

1 Voir, par exemple, Thomas Chapais, Cours d'histoire du Canada (8 vol., Québec, 1919-1934), IV: 8-9; Francis-J. Audet, Les députés de Montréal (ville et comtés), 1792-1867 (Montréal, 1943), 384-86; Helen Taft Manning, The Revolt of French Canada, 1800-1835 (Toronto, 1962), 356-57. Le même jugement se retrouve implicitement chez le biographe de Durham, Chester New, Lord Durham's Mission to Canada, édité par H. W. McCready (Toronto, 1963), ch. 1.

2 Audet, op. cit., fait remarquer que George-Étienne Cartier exprima ce point de vue à l'époque de la Confédération.

3 Voir, par exemple, Gérard Filteau, Histoire des Patriotes (3 vol., Montréal, 1938-1942), I: 166-72, et la recension signée par Lionel Groulx dans RHAF, 17 (1963-64): 291-293. Selon Filteau, les conceptions des Patriotes seraient un amalgame des usages britanniques et américains. Les conseillers de l'Exécutif auraient été responsables (dans un sens qui reste indéterminé) à l'Assemblée, mais n'y auraient pas siégé, non plus qu'au sein du Conseil législatif électif. C'est là, à vrai dire, à peu près le but que poursuivait Papineau (voir, ci-dessous, n. 31-34). 
On se propose, dans ce court article, d'exposer quelques considérations générales sur ces questions.

Les auteurs qui soulignent l'ignorance constitutionnelle des Patriotes citent souvent, pour justifier leur thèse, l'expulsion de Dominique Mondelet. Membre de l'Assemblée et patriote, celui-ci fut nommé au Conseil exécutif par lord Aylmer, en 1832. À la suite d'un acerbe débat, l'Assemblée déclara son siège vacant. La nomination au Conseil d'un simple membre de la majorité parlementaire était bien éloignée, pourtant, de la notion de la responsabilité ministérielle ou même de l'idée, préconisée au début du siècle par Pierre-Stanislas Bédard, d'un Conseil exécutif à deux composantes, qui eût été formé d'un groupe de conseillers du gouverneur et d'un groupe choisi parmi les leaders de la majorité à l'Assemblée ${ }^{4}$. Au cours des débats, à l'Assemblée, sur la nomination de Mondelet, ceux qui voulaient son expulsion exprimèrent l'opinion, non déraisonnable, qu'il ne serait guère plus que le commissionnaire du gouverneur, comme l'avait été avant lui Philippe Panet ${ }^{5}$. Loin de révéler une ignorance de la responsabilité ministérielle, les débats accrurent, en fait, l'intérêt pour cette question. Le 20 novembre 1832, un article parut dans la Quebec Gazette published by Authority, qui faisait remarquer que la nomination d'un «organ of the Executive in the Assembly» n'était sûrement pas la solution constitutionnelle idéale ${ }^{6}$. L'auteur de l'article expliquait ensuite l'usage suivi en Grande-Bretagne :

From the moment that the Government in England no longer commanded the supplies without a majority in the House of Commons, the Crown found it necessary to dispense with its former advisers and take the advice of parliamentary majorities. These advisers become responsible to Parliament for the acts of the Government; they are forced to conduct it comfortably [sic] to the views of the parliamentary majorities and were maintained themselves in Parliament and no longer [sic].

4 Le Canadien, 24, 31 janvier 1807; Manning, The Revolt, 70-71. Selon le projet de Bédard le gouverneur aurait eu, dans tous les cas, le loisir d'accepter ou de rejeter les avis.

5 La Minerve, 22, 26 novembre, 3 décembre 1832.

6 On doit distinguer cette publication officielle de celle de John Neilson, The Quebec Gazette - La Gazette de Québec. Les citations sont tirées de la réimpression faite par le Vindicator (voir note suivante). 
L'auteur recommandait que ce système fût adopté dans le BasCanada:

It is obvious that our Government cannot go on for any length of time, without a much greater approximation to the English practice. The principal offices of the Government must be held by men participating in the views of the parliamentary majorities... The attending to that business there, is in fact a part of the duties of their offices, which they must perform under the responsibility of losing them; and in a way to secure them the support of a majority... As things stand at the present, the Government of the Province is next to impossible: its consequences are anarchy or despotism...

Le Vindicator reproduisit ce texte au profit de ses lecteurs ${ }^{7}$ et $L a$ Minverve en donna une traduction française ${ }^{8}$, - mais aucun journal patriote ne commenta l'affaire. Deux semaines plus tôt, Étienne Parent avait toutefois, dans Le Canadien, pris fortement position en faveur de l'adoption du système britannique de la responsabilité ministérielle. Les habitants du Bas-Canada, écrivait-il, ne pourront jamais

...espérer de paix et de l'harmonie dans le gouvernement, tant que la constitution n'entourera pas le représentant du Roi d'hommes responsables de tous les actes administratifs, et jouissant de la confiance des Chambres, comme c'est le cas dans tout gouvernement représentatif bien organisé 9 .

Pendant une période d'environ trois ans, la question du gouvernement responsable resta dans l'ombre. Quand on s'y intéressa de nouveau, à la fin de 1835, ce fut apparemment sous l'influence d'événements qui se passaient dans le Haut-Canada, en particulier la publication, plus tôt la même année, du fameux Seventh Report... on Grievances ${ }^{10}$ de William Lyon Mackenzie. S'il préférait le système américain, avec ses gouverneurs élus, Mackenzie était néanmoins disposé par moments à s'accommoder d'un gouvernement responsable, - lequel était la solution de prédilection de ses alliés

723 novembre 1832 .

826 novembre 1832 .

97 novembre 1832. Voir aussi son éditorial du 19 juin 1832. On notera que Parent se réfère à un ministère qui jouit de la confiance «des Chambres». Il faut se rappeler que c'est en 1833 seulement qu'il fut définitivement établi, en Angleterre, que le ministère n'était aucunement responsable à la chambre des Lords.

10 W. L. Mackenzie, Seventh Report from the Select Committee of the House of Assembly of Upper Canada on Grievances (Toronto, 1835). 
réformistes plus modérés, William Warren et Robert Baldwin ${ }^{11}$. L'une des questions posées par Mackenzie à ceux dont il sollicita le témoignage au cours de la préparation du Seventh Report indiquait, comme l'a fait remarquer William Kilbourn, une solide connaissance du principe général de la représentation ministérielle au sein du gouvernement:

Would not the British Constitutional system by which the head of the government is obliged to choose his Councillors and principal officers from men possessing the Confidence of the popular branch of the Legislature, be more suitable to the wants and wishes of the country, if adopted in Upper Canada, than the present irresponsible mode of government ${ }^{12}$ ?

Le Report lui-même en arrivait à conclure qu'il y avait deux réformes possibles du pouvoir exécutif: soit des «elective institutions», soit «a responsible ministry... to hold office or lose it according as they may happen to be in minority or majority in the House of Assembly» ${ }^{13}$. Peu après la présentation de ce Report, l'Assemblée du Haut-Canada adopta une pétition au roi, dans laquelle elle approuvait le principe "[of the Crown] graciously consulting the wishes of your faithful people as expressed by their representatives in the choice of responsible advisers ${ }^{14}$.

À l'automne de 1835, Mackenzie eut l'occasion de faire part personnellement aux Patriotes de sa pensée, et sans doute de celle des Baldwin, sur la question constitutionnelle. Le 31 octobre, en compagnie d'un collègue réformiste, le $\mathrm{Dr}$ William O'Grady, il arrivait à Montréal pour y commencer un séjour de trois semaines dans le Bas-Canada. Officiellement, il y venait pour promouvoir les intérêts de la Welland Canal Company, dont il était un des directeurs, mais O'Grady et lui furent aussi reçus à titre de délégués des réformistes du Haut-Canada. La Minerve rapportait avec joie que « nos frères réformistes du Haut-Canada... sont prêts... à faire cause commune... sur les bases d'un gouvernement colonial responsable» ${ }^{15}$. À Québec, Mackenzie et son compagnon furent chaleu-

11 Voir New, Durham's Mission, 95-97.

12 Cité dans William Kilbourne, The Firebrand: William Lyon Mackenzie and the Rebellion in Upper Canada (Toronto, 1956), 117.

13 Cité dans New, Durham's Mission, 32.

14 Cité dans ibid.

is 5 novembre 1835 . 
reusement accueillis par les Patriotes: «All the liberal members have flocked around them to testify the sincere interest they take in the good cause in Upper Canada, and to tender them their most hearty co-operation. ${ }^{16}$

Il semble évident que l'un des moyens de collaboration, entre les deux groupes, fut de faire campagne conjointement en vue de l'obtention du gouvernement responsable. Au début de la session législative de 1835-1836, - Mackenzie était encore dans la capitale, - le secrétaire de la Commission Gosford, T. Frederick Elliott, notait que la faveur dont jouissait cette idée dans le Haut-Canada avait influencé la pensée du leader patriote Louis-Joseph Papineau ${ }^{17}$. Elliott informait aussi son correspondant du Colonial Office des opinions constitutionnelles d'un autre patriote, qu'il présentait comme "[o]ne of the first Members of the popular party in the House of Assembly ${ }^{18}$. Ce personnage lui avait dit

... that if, to the control which the Assembly was to have over all the Finances, were added a responsible Executive Council of Govt., he would desire no more, but would waive all particular Grievances and all demands of an Elective Legislative Council.

Et encore l'informateur d'Elliott n'était-il pas le seul à penser ainsi :

This project, of having three or five salaried Councillors, to be chosen from the leading men of the Colony, with Seats in the Legislature, and bound to get supplies or vacate their posts, seems to be fast gaining ground.

Le jugement d'Elliott se trouva confirmé dans une certaine mesure, au cours de la même session, quand, par la suite, l'Assemblée fit connaître officiellement sa position sur la question de la responsabilité ministérielle. Ce fut à l'occasion d'un débat provoqué par des remarques du lieutenant-gouverneur Colborne dans son discours du trône, prononcé devant l'Assemblée législative du HautCanada en janvier $1836^{19}$. Peu diplomatiquement, Colborne avait parlé de la «peculiar position of Lower Canada» et affirmé que les

16 Vindicator, 6 novembre 1835.

17 Elliott à Henry Taylor, 12 novembre 1835, RPAC (1883), 166-67. Cet extrait est cité ci-dessous (voir n. 29).

18 Ibid.

19 Vindicator, 22 janvier 1836. 
dissensions politiques qui y sévissaient «[had] tended to discourage Emigration and the transfer of capital to this country». Papineau et les Patriotes virent dans ces propos une tentative criante de diviser pour régner. Afin de bien montrer qu'à la vérité les réformistes étaient unis, une série de résolutions (adoptées par la suite) furent proposées par Edmund Bailey O'Callaghan, rédacteur du Vindicator et lieutenant de confiance de Papineau. Ces résolutions proclamaient, entre autres choses, que l'une des principales réformes que l'on désirait au Bas-Canada était «de rendre le Conseil Exécutif de cette Province directement responsable aux Représentants du Peuple conformément aux principes et à la pratique de la Constitution Britannique ${ }^{20}$. O'Callaghan se donna beaucoup de peine, dans son discours sur les résolutions, pour montrer que les «abuses and remedies thereunto proposed were precisely similar in both Provinces ${ }^{21}$. En parlant des défauts et de la réforme du pouvoir exécutif, O'Callaghan se référa au Seventh Report de Mackenzie. Il fit remarquer que le Conseil exécutif du Haut-Canada «was declared to be a non-descript in which they could place no confidence and agaisnt which it was useless to contend». La situation était la même dans la province d'en bas:

The individuals composing this body in Lower Canada, with small exception, have no power whatever individually; as a body they have that of doing harm and have used it in this sense with the greatest prodigality. The Governor is not obliged to submit his despatches to the Council or if he submits them he is not obliged to take their advice.

O’Callaghan présenta la solution privilégiée par les réformistes du Haut-Canada comme étant, en somme, une demande pour que le Conseil exécutif «should be responsible to the representatives of the people, as is the case in England». Le Bas-Canada, ajouta-t-il, veut les mêmes changements:

They simply demanded the rights of British subjects - that the British Constitution should be put into practice as it obtains

20 JHALC (1835-36), 15 février 1836, 474-76. L'Assemblée décida d'envoyer des copies des résolutions aux présidents des Assemblées des diverses colonies de l'Amérique du Nord britannique et exprima le désir «de coopérer cordialement avec les dites Assemblées dans toutes les mesures constitutionnelles propres à promouvoir les intérêts mutuels de ces Colonies.»

21 Vindicator, 23 février 1836. Les comptes rendus de La Minerve (29 février 1836) et du Canadien (22 février 1836) sont moins complets que ceux du Vindicator relativement à cette partie du discours d'O'Callaghan. 
in England - that as soon as the Executive ceased to agree with the majority of the House, it should resign in the same manner as the Ministry in England. Any person who heard him - at all acquainted with the workings of the British Constitution - could not but know that this was one of the main pillars upon which the system rests - that a minister cannot hold his place for one moment after he is in a minority. The same principles were demanded for this Province. The people wish to have some of the practical principles of the British Constitution applied to that «nondescript, against which it is folly to contend.»

Ainsi, à deux moments - novembre 1832 d'abord, fin de 1835 et début de 1836 ensuite - l'idée d'un gouvernement responsable pour le Bas-Canada fut discutée parmi les patriotes les plus influents. Il est vrai que la notion même de la responsabilité ministérielle fut alors exprimée avec moins de précision que ne le ferait Robert Baldwin dans sa célèbre lettre à lord Glenelg, en $1836^{22}$; il est vrai aussi qu'aucun patriote n'aborda la difficile question des relations entre le gouverneur et le conseil, ni la répartition des juridictions entre le pouvoir impérial et le pouvoir local ${ }^{23}$. Néanmoins, le principe essentiel du gouvernement responsable en ressort clairement: la nécessité pour les conseillers politiques de la Couronne de conserver l'appui de la majorité au sein de l'Assemblée, formée de membres élus. Et l'on peut ajouter que cette nécessité fut parfois présentée avec un corollaire: l'obligation constitutionnelle stricte de démissionner, advenant qu'ils perdissent la confiance de l'Assemblée, - proposition délicate que ni lord Durham ni son secrétaire Charles Buller ne préconisèrent ${ }^{24}$.

22 Baldwin à Glenelg, 13 juillet 1836, RPAC (1923), 329-37.

23 La première formulation, clairement énoncée, des rapports du gouverneur avec son Conseil exécutif responsable se trouve dans une lettre de lord Grey au lieutenant-gouverneur John Harvey, de la Nouvelle-Écosse, 3 novembre 1846, W.P.M. Kennedy, Documents of the Canadian Constitution, 1759-1915 (Toronto, 1918), 570-73. C'est lord Durham qui, le premier, préconisa une division des juridictions entre le pouvoir impérial et le pouvoir local (New, Durham's Mission, 178-80).

24 Durham [Sir Charles Lucas, ed., Lord Durham's Report (3 vol., Oxford, 1912), II : 279-81] et Buller [E.M. Wrong, Charles Buller and Responsible Government (Oxford, 1926), passim] étaient tous deux disposés, dans les circonstances exceptionnelles, à permettre au gouverneur de maintenir - en dépit des pressions politiques - un Conseil exécutif appuyé seulement par une minorité. L'usage constitutionnel selon lequel un gouvernement défait lors d'un vote de confiance doit démissionner sur-le-champ (comme l'affirmait O'Callaghan) ou demander la dissolution ne devait s'imposer que par la suite. 
Cela étant dit, il importe de remarquer que la responsabilité ministérielle ne fut jamais au premier plan dans la pensée constitutionnelle des Patriotes, en ce sens qu'elle ne fut jamais considérée sur le même pied que la demande d'un conseil législatif à caractère électif, et encore moins comme une réforme sans laquelle tout autre changement d'ordre constitutionnel serait inutile (comme le pensaient les Baldwin). Les Quatre-vingt-douze résolutions de 1834 consacraient des douzaines de paragraphes au Conseil législatif existant et à celui que l'on proposait ${ }^{25}$. Une seule fois on fit mention du Conseil exécutif, et ce fut seulement pour se plaindre de «[its] vicious composition and irresponsibility " ${ }^{26}$. Même en 1836 l'idée de la responsabilité ministérielle n'était pas particulièrement dans l'air. Le 26 février, l'Assemblée adopta des adresses au roi et aux deux chambres du Parlement, dans lesquelles on traitait une fois de plus, et en détail, de la question d'un conseil législatif à caractère électif. La seule mention qu'on y faisait au Conseil exécutif était à la fois brève et assez vague:

Au sujet du Conseil Exécutif, nous nous abstiendrons d'entrer dans aucuns détails... Nous nous bornerons à dire que la reconnaissance pleine et entière des droits de cette Chambre et de ceux du Peuple par ceux qu'il plaira à Votre Majesté d'appeler à ses Conseils et leur responsabilité Constitutionnelle basée sur la Pratique du Royaume-Uni, seront des motifs essentiels de confiance dans le Gouvernement de Votre Majesté ${ }^{27}$.

Au cours des assemblées populaires contre les Résolutions Russell, pendant l'été et l'automne de 1837, on parla bien peu, si jamais on le fit, de la responsabilité ministérielle ${ }^{28}$.

De fait, on possède des indications précises que, en 1835 et en 1836, la réforme possible d'un gouvernement responsable était perçue, par Papineau et O'Callaghan à tout le moins, comme une concession qu'ils faisaient à contrecœur aux réformistes du HautCanada, plutôt que comme une idée qui valait d'être adoptée pour elle-même. Dans la lettre d'Elliott déjà citée, le secrétaire de la

25 Kennedy, Documents, 366-88.

26 Ibid., 385 (résolution 84).

27 JHALC (1835-36), 26 février 1836, 548.

28 Du moins selon les rapports du Vindicator et de La Minerve. Une fois O'Callaghan se plaignit de la nomination de deux «bureaucrates» au sein du Conseil exécutif, en faisant valoir que «the confidence of the public, expressed by the Representative branch of the Legislature is wanting...» (Vindicator, 3 octobre 1837). 
Commission Gosford en venait à écrire, relativement à un conseil exécutif responsable:

Papineau dislikes it, because he knows it would put a bit in his mouth, but he confessed to me the other day, that from the strong feeling in its favour in the Assembly, and from the approval of the same object among the popular party in Upper Canada, with whom he is very desirous to co-operate, he should be disposed not to press his Individual opposition to the scheme ${ }^{29}$.

Écrivant à Papineau en 1844, O'Callaghan lui rappela que:

... I looked with strong feelings of prejudice at the movement which the Reformers of Upper Canada forced on us of Lower Canada in 1835,6 in favor of their peculiar hobby «Responsible Government", which I felt strongly inclined to object to, at that time, but abstained from doing for the sake of harmony ${ }^{30}$.

Il n'est pas difficile d'imaginer les raisons de la méfiance que, parmi les principaux chefs patriotes, - qui réglaient leurs sentiments sur ceux de Papineau, - on éprouvait pour le gouvernement responsable. Dans le domaine constitutionnel, Papineau, depuis longtemps, considérait avec suspicion tout ce qui était britannique. Quand, enfin, viendrait l'indépendance, - comme inévitablement elle devait venir, selon lui, - on aurait une république avec, à sa tête, un président. En attendant ce jour, Papineau semble avoir cru possible une maîtrise effective du pouvoir exécutif, à la condition que trois réformes fussent acquises: autorité réelle de l'Assemblée sur les finances du gouvernement ${ }^{31}$, conseil législatif élu, et pouvoir conféré à ce dernier de juger les mises en accusation (impeachments) - de conseillers de l'Exécutif et de fonctionnaires - émanées de l'Assemblée. Si l'on accordait un conseil législatif à caractère électif qui eût le pouvoir de se prononcer sur les mises en

29 Elliott à Taylor, 12 novembre 1835 (voir, ci-dessus, n. 17).

30 O'Callaghan à Papineau, 17 juillet 1844, APC, M.G. 24, B. 2 (Papiers Papineau), 3: 3993-98. A titre de corroboration partielle, on peut noter que O'Callaghan publia une brochure, à la suite de la session au cours de laquelle, sur une motion de sa part, l'Assemblée se déclara en faveur du gouvernement responsable. La brochure [The Late Session of the Provincial Parliament in Lower Canada (Montréal, 1836)] ne fait pas mention, toutefois, de griefs contre le pouvoir exécutif; l'auteur fait porter toute son attaque contre le Conseil législatif, qui avait rejeté, ignoré ou modifié notablement quarante-neuf des cent sept projets de loi que lui avait transmis l'Assemblée.

31 C'est-à-dire que les gouverneurs ne pourraient pas rendre ineffectives une coupure ou une diminution des crédits en ayant recours à des fonds de l'armée. 
accusation, selon ce qu'il dit à sir George Gipps, l'un des membres de la Commission Gosford, Papineau pourrait bien consentir à examiner la question d'une liste civile qui couvrirait les salaires de quelques-uns des hauts fonctionnaires. Le conseil électif, affirmait-il, ferait pencher d'une façon décisive, du côté de l'Assemblée plutôt que du gouverneur, la balance du pouvoir; et le gouverneur serait amené à faire preuve d'intelligence et d'honnêteté dans ses nominations à la fonction publique ${ }^{32}$. Ainsi Papineau paraît-il avoir estimé que, tout comme aux Etats-Unis, les deux pouvoirs, exécutif et législatif, devaient être séparés, mais que le législatif devait disposer de moyens réels, grâce au contrôle des finances et à la soumission des mises en accusation devant une chambre haute élective, pour réprimer les abus de l'exécutif ${ }^{33}$. Sans doute, aussi, était-il confiant que l'Assemblée, par ses comités, serait capable de surveiller de près les divers domaines de l'administration publique, de la même façon qu'elle le faisait déjà selon les dispositions de la loi scolaire de $1829^{34}$.

Un deuxième motif de défiance fut vraisemblablement la possibilité (dont il arrivait à Mackenzie de s'inquiéter ${ }^{35}$ ) que la responsabilité ministérielle ne présentât qu'une solution partielle ou apparente, qui éroderait la volonté de réclamer avec insistance la reconnaissance, relativement, en particulier, à la chambre haute, du principe électif.

32 Papineau à 'John Arthur Roebuck, 23 octobre 1835, APC, M.G. 24, A. 19 (Papiers John Arthur Roebuck), 1, 5: 37-42.

33 Pour montrer qu'il était sympathique au projet d'un gouvernement responsable, Groulx (voir n. 3) cite le passage suivant d'une lettre de Papineau à sa femme, du 28 janvier 1836: "... le seul principe essentiel est le contrôle absolu de la Représentation sur le revenu et son influence prépondérante et directe sur tous les actes de l'Exécutif.» Mais ce passage est trop peu précis au sujet des modalités d'application de ce "principe" pour être considéré comme une preuve que c'est à un gouvernement formé de ministres responsables que pensait Papineau. En août 1837, M. de Pontois, l'embassadeur de France aux États-Unis, tira d'une entrevue avec Papineau, à Montréal, une interprétation de la réforme proposée de l'Exécutif qui laisse croire que Papineau n'envisageait pas l'instauration d'un gouvernement doté d'un tel cabinet. Les Patriotes, écrivait de Pontois à son ministre des Affaires Étrangères (9 août 1837), demandent que «le Conseil Exécutif soit composé de personnes nées dans le Pays et non revêtues d'emplois du Gouvernement" (R. LaRoque de Roquebrune, éd., "M. de Pontois et la rébellion des Canadiens français en 1837-38 ", Nova Francia, 3 (1927-1928): 246-49.)

34 Louis-Philippe Audet, Le système scolaire de la Province de Québec (6 vol. Québec, 1950-1956), V: 161-74.

${ }_{35}$ V.g. Mackenzie à John Neilson, 22 février 1836, RPAC (1918), 64. 
Dernier motif, enfin, et peut-être le plus important des trois: on ne pouvait, en ces années qui marquaient en Grande-Bretagne le début de l'évolution du gouvernement fondé sur la responsabilité ministérielle, savoir avec certitude quels pouvoirs la Couronne pourrait se réserver. Le gouverneur utiliserait-il le système pour multiplier les «Chouayens», ainsi que les Patriotes désignaient communément Philippe Panet (1831) et Dominique Mondelet, que lord Aylmer avait nommés au Conseil exécutif? Ou bien un gouverneur ne révoqueraitil pas purement et simplement un conseil qui n'aurait pas l'heur de lui plaire, comme William IV avait congédié le gouvernement Melbourne en 1834 ?

Papineau, dès lors, eût peut-être mené campagne en faveur de la responsabilité ministérielle, si elle avait été intégrée aux autres réformes qu'il réclamait; mais, présentée comme solution unique, à la manière des Baldwin, il y était entièrement opposé. On trouve une indication intéressante de cette attitude dans une lettre à l'agent de la Chambre d'assemblée au Parlement, le député radical du parti Whig John Arthur Rœbuck ${ }^{36}$. Cette lettre fut écrite dans l'atmosphère d'extrême tension provoquée par la divulgation des instructions restrictives adressées à la Commission Gosford, mais il n'y a guère à douter que Papineau ait entretenu, précédemment, de semblables opinions ${ }^{37}$. Il expose d'abord ses craintes que Edward Ellice, membre important du parti Whig au Parlement et seigneur de Beauharnois, ne soit en train de comploter contre les Patriotes et les réformistes du Haut-Canada:

... j'ai bonne raison de croire que ce Mr. Ellice... profit[e] de ses liaisons dans le haut-Canada où il a la réputation de libéral, pour bien persuader de ne pas insister sur la demande du Conseil Electif, mais de se restreindre à celle du Conseil Exécutif responsable.

\footnotetext{
37 Voir ci-dessus, n. 29, la citation d'Elliott. Vraisemblablement, les désillusions des Patriotes à l'endroit de lord Gosford, - et des gouverneurs en général, - ensuite de la révélation de ses instructions, explique-t-elle le fait que Papineau minimise l'importance de l'objectif que représentait le gouvernement responsable (voir, ci-dessus, n. 28). Dans la lettre précédemment citée (n. 30), O'Callaghan parlait des années 1835 et 1836 , mais - et cela est significatif - il ne mentionnait point l'année 1837.
} 93-94.

36 Papineau à Roebuck, 9 mai 1836, Papiers Roebuck (voir, ci-dessus, n. 32), 
Accepter cela serait un marché de dupes:

Il [Ellice] offre l'impossible. Un Gouverneur nommé par Downing street, pour promouvoir ses créatures aux emplois, - pour faire réussir des projets sinon Anti du moins extra - Coloniaux... avec un Conseil Exécutif responsable sont des contradictions dans les termes.

À la faveur d'un tel système, les conseillers exécutifs deviendraient quasi inévitablement des «Chouayens»:

Ce plan ferait des hypocrites qui professeraient le libéralisme mais parce qu'ils seront en petit nombre agissant dans le secret, ils seront facilement gagnés.

Bien plus, poursuit Papineau, la constitution britannique, avec son caractère aristocratique et monarchique, ne convient décidément pas à l'Amérique du Nord «démocratique»:

C'est ce qu'ont bien compris les hommes d'État des anciennes Colonies, puisque quand ils auraient pu la conserver après avoir acquis leur indépendance ils l'ont répudiée pour en adopter une meilleure pour les Américains situés comme nous le sommes; quoiqu'elle fut peut être la moins bonne pour les Européens situés comme ils sont.

Si les réformistes du Haut-Canada entretiennent l'idée du gouvernement responsable, c'est simplement parce qu'ils sont effrayés, pour des raisons politiques, «d'avouer... que les institutions de leurs voisins sont bien justement les seules qui leur conviennent». L'influence corruptrice du gouvernement du Haut-Canada est telle que ces réformistes même qui sont en faveur d'un Conseil législatif à caractère électif (comme Mackenzie) ont dû temporiser, ayant

...cru plus prudent de faire de la question du Conseil Exécutif responsable l'objet apparent de leur difficulté avec le Gouvernement provincial. Mais la masse et les hommes influens parmi eux sentent bien que cette concession ne serait qu'un palliatif insuffisant. Ils ont demandé le Conseil Électif, mais plus faiblement à mon avis qu'ils n'auraient du le faire.

On ne peut que conjecturer sur ce qu'il serait advenu si les Patriotes avaient fait de la responsabilité ministérielle la pièce mâ̂tresse de leur programme constitutionnel, à l'instar des Baldwin dans le Haut-Canada. Vu son caractère britannique, et l'idée en eût-elle été adroitement mise de l'avant pendant un certain nombre 
d'années, la réforme eût peut-être obtenu quelque appui au Parlement. Dans les meilleures conditions, toutefois, les difficultés eussent été formidables ${ }^{38}$. De tous les ministres du parti Whig, dans les années 1830, seul lord Howick semble avoir caressé l'idée de doter le Canada d'un gouvernement responsable ${ }^{39}$. Politiquement, les Whigs ne pouvaient donner l'impression d'abandonner la minorité anglaise, qui eût sans aucun doute réagi bruyamment, et peutêtre même avec violence, à la moindre apparence qu'une telle réforme pût être réalisée. Sans compter, comme le démontra le rejet par lord John Russell de cette forme de gouvernement, dans la mesure où on voulait la mettre en œuvre dans une colonie, que les notions de responsabilité ministérielle et de souveraineté impériale n'étaient pas, dans ce contexte, aisément conciliables. Antérieurement au triomphe de la liberté du commerce, à la fin des années 1840, et à la montée du sentiment de la «little England», les gouvernements du parti Whig n'étaient simplement pas disposés à mettre en danger le lien impérial. Peut-être n'existait-il aucune issue pacifique à l'impasse constitutionnelle des années 1830 , hors l'abandon par les Patriotes de leur désir de voir l'Assemblée mâltresse du pouvoir.

(Traduction: André Vachon, s.r.c.)

38 Voir O.A. Kinchen, Lord Russell's Canadian Policy (Lubbock, Texas, 1945); Helen Taft Manning, "The Colonial Policy of the Whig Ministers, 1830-37", CHR, 33 (1952), 203-36, 341-68; Peter Burroughs, The Canadian Crisis and British Colonial Policy, 1828-1841 (Toronto, 1972).

39 Burroughs, Canadian Crisis, 75; Elliott à Taylor, 12 novembre 1835 (voir, ci-dessus, n. 17). 